\title{
Oferta de gêneros alimentícios provenientes da agricultura familiar em escolas municipais de Goiânia - GO
}

\author{
Suministro de productos alimenticios de la agricultura familiar en escuelas de Goiânia - \\ GO municipales
}

Supply of food from family farms in municipal schools in Goiânia-GO

lel Marciano de Moraes Filho ${ }^{*}$, Laylla Luanna de Mello Frasca ${ }^{2}$, Ludmilla Santos de Almeida ${ }^{3}$, Maria Fernanda Rocha Proença ${ }^{1}$, Rodrigo Marques da Silva ${ }^{1}$, Osmar Pereira dos Santos ${ }^{4}$, Keila Cristina Félis $^{5}$, Eloiza Helena Carrijo Barbosa ${ }^{3}$.

\section{RESUMO}

Objetivo Avaliação da oferta de gêneros alimentícios provenientes da Agricultura Familiar em escolas municipais de Goiânia. Método: Trata-se de um estudo observacional descritivo, desenvolvido em Instituições Educacionais Municipais da cidade de Goiânia-Goiás. Foram visitadas 6 unidades escolares da Região Central. Para a realização da avaliação do cardápio aplicou-se o método de Avaliação Qualitativa das Preparações de Cardápio e para avaliar a frequência dos alimentos fornecidos pela Agricultura Familiar aplicou-se um checklist incluindo os tipos e a frequência de alimentos ofertados. As análises foram realizadas no software Stata 8.0. Resultados: Constatou-se que o percentual obtido para o consumo de frutas, folhosos e verduras fora baixo. Ao analisar os alimentos provenientes da Agricultura Familiar, notou-se que as frutas mais consumidas foram abacaxi e mamão. Verificou-se que a bebida láctea, o queijo do tipo muçarela e o requeijão cremoso representam a porcentagem total de oferta. Conclusão :Conclui-se, portanto, que a oferta de frutas e verduras são baixos, provavelmente por questões ligadas a distribuição, o que pode ser adequado com a inclusão em maior frequência de alimentos provenientes da Agricultura Familiar. Para que assim evite de maneira inerente os possíveis agravos da alimentação inadequada na vida adulta destas crianças.

Palavras-chaves: Alimentação Escolar, Consumo de Alimentos, Agricultura Sustentável.

\begin{abstract}
Objective of this study was to evaluate the supply of foodstuffs from Family Agriculture in municipal schools in Goiânia. Method: This is a descriptive observational study, developed in Municipal Educational Institutions of the city of Goiânia-Goiás. Six school units from the Central Region were visited. For the evaluation of the menu the method of Qualitative Evaluation of the Menu Preparations was applied and to check the frequency of the food provided by the Family Agriculture a checklist was applied including the types and the frequency of foods offered. The analyzes were performed in Stata 8.0 software. Results: It was verified that the percentage obtained for the consumption of fruits, leafy vegetables and was low. When analyzing food from Family Agriculture, it was noticed that the fruits most consumed were pineapple and papaya. It was verified that the milk drink, the mozzarella cheese and the creamy curd represent the total percentage of supply. Conclusion: It is concluded, therefore, that the supply of fruits and vegetables are low, probably due to distribution issues, which may be adequate with the inclusion of food from Family Farming. thus avoiding inherently the possible aggravations of inadequate feeding in the adult life of these children.
\end{abstract}

Keywords: School Feeding, Food Consumption Sustainable Agriculture.

\footnotetext{
${ }^{1}$ Faculdade Sena Aires (Facesa) - Valparaiso de Goiás, Goiás. *E-mail: ielfilho@yahoo.com.br

2 Universidade Federal de Goiás (UFG) - Goiânia, Goiás.

${ }^{3}$ Pontifícia Universidade Católica de Goiás (PUC-GO) - Goiânia, Goiás.

${ }^{4}$ Faculdade União de Goyazes (FUG) - Trindade, Goiás.

${ }^{5}$ Centro Universitário de Goiatuba (UniCerrado). Goiatuba, Goiás.
} 


\section{RESUMEN}

El objetivo del estudio fue evaluado la oferta de géneros alimenticios provenientes de la Agricultura Familiar en escuelas municipales de Goiânia. Método: Se trata de un estudio observacional descriptivo, desarrollado en Instituciones Educativas Municipales de la ciudad de Goiânia-Goiás. Se visitaron 6 unidades escolares de la Región Central. Para la realización de la evaluación del menú se aplicó el método de Evaluación Cualitativa de las Preparaciones de menú y para evaluar la frecuencia de los alimentos suministrados por la Agricultura Familiar se aplicó un checklist incluyendo los tipos y la frecuencia de alimentos ofertados. Los análisis se realizaron en el software Stata 8.0. Resultados: Se constató que el porcentaje obtenido para el consumo de frutas, folios y verduras fuera bajo. Al analizar los alimentos provenientes de la Agricultura Familiar, se notó que las frutas más consumidas fueron piña y papaya. Se comprobó que la bebida láctea, el queso de tipo mozzarella y el requesón cremoso representan el porcentaje total de oferta. Conclusión: Se concluye, por lo tanto, que la oferta de frutas y verduras es baja, probablemente por cuestiones ligadas à la distribución, lo que puede ser adecuado con la inclusión en mayor frecuencia de alimentos provenientes de la Agricultura Familiar. así evitando de manera inherente los posibles agravios de la alimentación inadecuada en la vida adulta de estos niños.

Palabras claves: Alimentación Escolar, Consumo de alimentos, Agricultura Sostenible.

\section{INTRODUÇÃO}

A agricultura familiar para o cidadão é um mecanismo de conquistas no campo da segurança e soberania alimentar, corroborando com a proteção da agro biodiversidade, logo a mesma pode ser considerada, como um instrumento para o uso sustentável dos recursos naturais e valorização de alimentos tradicionais e preservação da pluralidade cultural, visando uma oportunidade de impulsionar as economias locais (FAO, 2014).

Entre essas associações enquadra-se a alimentação escolar, devido a presença de alimentos produzidos no ambiente escolar, por meio da sua inserção nos cardápios do Programa Nacional de Alimentação Escolar (PNAE) (TEO CRPA, et al., 2017). O PNAE, instituído inicialmente em 1955, atribuiu atender às necessidades nutricionais dos alunos durante a sua permanência no ambiente escolar, contribuindo assim para o crescimento, desenvolvimento, processo de aprendizagem e memorização e rendimento escolar. O mesmo também opera como uma maneira de promover a formação de hábitos alimentares saudáveis, por meio da oferta da alimentação escolar e de ações educacionais na alimentação nutricional, empregada a todas faixas etárias da educação básica de caráter público (BRASIL, 2017).

De acordo com a resolução no 38 , de 8 de outubro de 2013, e do Fundo Nacional do desenvolvimento da educação (FNDE), assegura e estabelece orientações e procedimentos para o pagamento de bolsas de estudo e pesquisa no âmbito da Escola da Terra, em consonância com a Lei no 11.947 de 16 de junho de 2009, as escolas públicas passaram a consumir os produtos da agricultura familiar em suas refeições oferecidas nos ambientes escolares (SCHRÖETTER MR, 2011).

Atualmente, de acordo com a Resolução no 26/2013, o governo federal repassa, aos estados, municípios e escolas federais, valores financeiros de caráter suplementar, atribuídos ao número de alunos matriculados no ano anterior em cada rede de ensino, para serem utilizados na aquisição de gêneros da alimentação escolar. Contudo, no final de cada ano realiza-se a prestação de contas pelo município, visando que a gestão se dá de forma centralizada. Diante do pressuposto parte-se do conhecimento das práticas alimentares participam de processos identitários, devido ao papel central que a alimentação assume no aprendizado e na formação social. Por sua natureza vital, rotineira, geradora de sociabilidades e possibilidades de escolhas (SILVA EO, et al., 2018).

A alimentação escolar assume papel importante pelo potencial apresentado na reconfiguração do perfil alimentar e nutricional da população brasileira, especialmente por ser na infância e na adolescência que se estabelecem mais fortemente os hábitos alimentares, sendo considerado fundamental a variedade, quantidade e a adequação destas refeições (TEO CRPA, et al., 2017). 
Adicionalmente, o conhecimento da situação nutricional é essencial na avaliação das condições de saúde da população, além de entender os comportamentos e as atitudes alimentares das crianças. Alimentos provenientes da agricultura familiar nas práticas alimentares, valoriza os alimentos regionais, aumenta 0 consumo de hortaliças, atua em práticas sustentáveis e gera renda, principalmente no meio rural (OLIVEIRA $R G, 2015)$.

Portanto, o objetivo desse trabalho é apresentar uma análise dos cardápios da alimentação escolar ofertados aos alunos de rede pública municipal de Goiânia, quanto a oferta de frutas, hortaliças, alimentos processados oriundos da agricultura familiar.

\section{MATERIAL E MÉTODOS}

O experimento, de natureza observacional descritivo, foi executado em Instituições Educacionais Municipais, localizadas no município de Goiânia-GO. Tais instituições eram divididas por nove regiões, sendo elas: Região Mendanha; Noroeste, Vale do Meia Ponte, Sudoeste, Oeste, Central, Leste, Sul e Norte. A pesquisa foi descrita em seis escolas localizadas na Região Central, em que todas atribuem ao seu cardápio produtos alimentícios oriundos da agricultura familiar. Cada escola recebeu um Termo de Consentimento Livre e Esclarecido, informado com uma linguagem clara sobre natureza da pesquisa, seus objetivos, justificativas e procedimentos.

A avaliação realizada no cardápio das escolas, foi seguido pelo o método de Avaliação Qualitativa das Preparações do Cardápio (AQPC). Tal avaliação ocorreu no cardápio do mês de setembro de 2015, em dias úteis, totalizando 19 dias.

As variáveis analisadas foram: o processo de cocção, a coloração dos preparos e dos alimentos empregados no cardápio, as fritura que se apresentaram de maneira isolada, acompanhamento de doces, a constância de frutas ou doces (preparações pré-prontas que contêm açúcar como protagonista de seus ingredientes, como bebidas lácteas e achocolatados, refresco artificial, creme, canjica e bolos), presença de verduras e folhosos, o aparecimento de conservas, saladas, presença de alimentos ricos em enxofre e oferta a de carne gordurosa (ALMEIDA L, et al., 2018).

A análise dos dados foi realizada de acordo com as observações do percentual de ocorrência diária dos alimentos ou preparações relativas a cada critério. Em seguida, foi contabilizado por semana o número de dias da ocorrência de cada parâmetro analisado. Contudo, calculou-se a porcentagem de ocorrência, considerando todos os dias do mês. A avaliação da frequência dos alimentos fornecidos pela agricultura familiar, aplicou-se um checklist incluindo os tipos e a frequência de alimentos ofertados. Tal identificação era realizada pelas notas fiscais referente ao mês de coleta.

O trabalho foi submetido ao Comitê de ética pesquisa da Pontifícia Universidade Católica sobre o número 35541 na data 20/06/2012 de Goiás e o parecer foi: O projeto não necessita ser avaliado pelo CEP por não lidar diretamente com pessoas e estar embasado em dados observacionais. Basta que a proponente obtenha permissão das escolas para observar suas rotinas.

Os dados foram submetidos a análise descritiva, utilizando-se a frequência absoluta e relativa, média e desvio padrão, mínimo e máximo. As análises foram realizadas no software Stata 8.0.

\section{RESULTADOS}

A compilações descritiva dos dados da Análise Qualitativa das preparações do cardápio das escolas municipais localizadas na Região Central, demonstrou variações entre as escolas. A escola $F$ obteve melhores ocorrências nos parâmetros avaliados, as demais escolas não diferiram entre si (Tabela 1).

Analisando cada parâmetro avaliado, podemos observar que o percentual obtido na análise dos cardápios da escola, houve diversas porcentagens de ocorrência. O consumo de frutas estabeleceu $21,9 \%, 44.7 \%$ nas folhagens e verduras, $0 \%$ em salada de conserva, $7.8 \%$ na semelhança entre as cores da preparação 
oferecida no cardápio, $0 \%$ em alimentos ricos em enxofre, 33,3\% de carnes gordurosas, $0 \%$ de frituras, $62,2 \%$ de doces e $0 \%$ na associação de doces e frituras (Tabela 1).

Tabela 1 - Análise Qualitativa das Preparações do Cardápio das escolas municipais localizadas na Região Central. Goiânia, Goiás, 2012.

\begin{tabular}{cccccccccc}
\hline Escolas & $\begin{array}{c}\text { Dias de } \\
\text { cardápio }\end{array}$ & Frituras & Fruta & Folhosos & $\begin{array}{c}\text { Cores } \\
\text { iguais }\end{array}$ & $\begin{array}{c}\text { Ricos em } \\
\text { enxofre }\end{array}$ & $\begin{array}{c}\text { Carne } \\
\text { gordurosa }\end{array}$ & Doce $\begin{array}{c}\text { Doce } \\
+ \\
\text { Fritura }\end{array}$ \\
\hline A & 19 & - & 3 & 5 & 1 & - & 5 & 9 & - \\
B & 19 & - & 3 & 5 & 1 & - & 5 & 9 & - \\
C & 19 & - & 3 & 5 & 1 & - & 5 & 9 & - \\
D & 19 & - & 3 & 5 & 1 & - & 5 & 9 & - \\
E & 19 & - & 3 & 5 & 1 & - & 5 & 9 & - \\
F & 19 & - & 10 & 26 & 4 & - & 13 & 26 & - \\
$\begin{array}{c}\text { Total de } \\
\text { dias }\end{array}$ & 114 & 0 & 25 & 51 & 9 & 0 & 38 & 71 & 0 \\
$\begin{array}{c}\text { Ocorrência } \\
\text { (\%) }\end{array}$ & 0 & 0 & 21,9 & 44,7 & 7,8 & 0 & 33,3 & 62,2 & 0 \\
\hline
\end{tabular}

Fonte: Dados da pesquisa, 2014.

As análises dos alimentos provenientes da Agricultura familiar destacam-se abacaxi, mamão, bebida Láctea, queijo muçarela, requeijão cremoso, manteiga de leite e polpa de frutas (Tabela 2). Os alimentos de maiores ocorrências são bebida láctea, queijo muçarela e requeijão cremoso, destaca-se nas seis escolas. A polpa de fruta estabelece menor distribuição oriunda da agricultura familiar, permanecendo somente em uma escola. Quanto ao recebimento mensal a polpa de frutas estabelece maiores médias totalizando quatro recebimentos mensais. Enquanto os demais alimentos exibem uma média de dois recebimentos ao mês. A frequência dos alimentos nos cardápios da s escolas, exibe uma variedade nos dias analisados, sendo a queijo muçarela a maior frequência e a bebida láctea e polpa de frutas menor frequência.

Tabela 2 - Alimentos presentes no cardápio provenientes da Agricultura Familiar.

\begin{tabular}{lccc}
\hline \multicolumn{1}{c}{ Alimentos } & $\begin{array}{c}\text { Frequência de escolas } \\
\mathbf{N}(\%)\end{array}$ & $\begin{array}{c}\text { Recebimento mensal } \\
\text { Média } \pm \text { DP } \\
\text { (mín-máx) }\end{array}$ & $\begin{array}{c}\text { Frequência no cardápio } \\
\text { Média } \pm \text { DP } \\
\text { (mín-máx) }\end{array}$ \\
\hline Abacaxi & $5(83,33)$ & $1,2 \pm 0,4(1-2)$ & $1,8 \pm 2,0(1-6)$ \\
Mamão & $5(83,33)$ & $1,2 \pm 0,4(1-2)$ & $1,8 \pm 2,0(1-6)$ \\
Bebida Láctea & $6(100,00)$ & $1,2 \pm 0,4(1-2)$ & $1,2 \pm 0,4(1-2)$ \\
Queijo muçarela & $6(100,00)$ & $1,8 \pm 0,8(1-3)$ & $2,2 \pm 2,9(1-8)$ \\
Requeijão cremoso & $6(100,00)$ & $1,2 \pm 0,4(1-2)$ & $1,8 \pm 2,0(1-6)$ \\
Manteiga de leite & $2(33,33)$ & $1,5 \pm 0,7(1-2)$ & $2 \pm 0$ \\
Polpa de fruta & $1(16,67)$ & $2 \pm 0$ & $4 \pm 0$
\end{tabular}

Fonte: Dados da pesquisa, 2014. 


\section{DISCUSSÃO}

O estudo evidenciou que o processo de aquisição de alimentos da agricultura familiar para a alimentação escolar não seguia grande parte das recomendações governamentais. Observou que oferta de frutas era como sobremesa é era baixo (21,9\%). Neutzling RAH et al. (2009) demonstram que o consumo de frutas, legumes e verduras tem sido associado à redução do risco de mortalidade e diminuição da ocorrência de doenças crônicas, tais como as doenças cardiovasculares, derrames e alguns tipos de câncer, dessa forma, aponta a importância do consumo desses alimentos diariamente. Segundo o Instituto Brasileiro de Geografia e Estatística (IBGE) no ano de 2014 as regiões sudeste e centro oeste registram os melhores índices de consumo de frutas e hortaliças, entre $42,8 \%$ e $43,9 \%$ respectivamente em comparação ao território nacional do Brasil. Aos contrários dos dados analisados no estudo em questão.

A ingestão de folhosos e verduras é essencial para se manter a saúde, são fontes de vitaminas, minerais e fibras e aparentemente desempenham um efeito protetor sobre o risco de obesidade, diabetes mellitus tipo II, doenças cardiovasculares e alguns tipos de câncer (HE FJ, et al., 2006). A Organização Mundial da Saúde (OMS) estima que cerca de 2,7 milhões de mortes por ano em todo o mundo sejam decorrentes do consumo insuficiente desses alimentos (LOCK K, et al., 2005).

Os programas de alimentação saudável das Diretrizes Nacionais se preocupam com a prevenção de doenças relacionadas à alimentação e nutrição. Neste caso, a má alimentação contribui para a desnutrição e prevalência de sobrepeso, obesidade e doenças crônicas não transmissíveis. O consumo alimentar é caracterizado pela capacidade em que os alimentos atendem as necessidades fisiológicas do consumidor. Os efeitos do desenvolvimento da criança e de todos os indivíduos são positivos quando os mesmos se alimentam de forma saudável. Por outro lado, a má alimentação, torna-se efeito de fatores sociais como fome, desnutrição, obesidade, mobilidade e mortalidade (SOUZA LCA, et al., 2019).

Conforme o Guia Alimentar para a População Brasileira uma alimentação saudável se configura na apresentação de uma ampla variedade de grupos alimentares com diferentes colorações e quanto mais colorida é a alimentação, mais rica é em termos de vitaminas e minerais. Sabe-se que o jogo das cores são atrativos muito utilizado para se obter uma alimentação prazerosa, contemplando também as recomendações nutricionais de um prato diversificado e colorido (PROENÇA RCP, et al., 2005; BRASIL, 2014).

Deve-se atentar para a presença de alimentos ricos em enxofre no cardápio por causarem desconforto abdominal e flatulência o que pode reduzir o rendimento dos alunos após a ingestão. Veiros (2005) verificou alta presença destes alimentos no cardápio, em $65 \%$ dos dias analisados em um restaurante, os comensais reclamavam bastante do desconforto após o almoço e Passos ALA (2008) também encontrou elevada quantidade, $57,7 \%$ dos dias. As recomendações feitas pelo o Guia Alimentar para a População Brasileira sugere reduzir a obtenção de energia proveniente das gorduras, com ênfase na diminuição do consumo do grupo das carnes vermelhas, ovos e produtos lácteos, como leite e queijos, sendo esses, alimentos ricos em gordura saturada (BRASIL, 2014).

A oferta de doces, principalmente formulados foi alta $(62,2 \%)$ isso pode ser devido ao baixo custo e maior aceitabilidade entre os alunos. Barbosa RMS et al. (2007), verificou que a ingestão de açúcar foi três vezes maior que o preconizado pela pirâmide alimentar infantil proposta por Philippi ST et al. (2003). Em um outro estudo realizado por Aquino RC e Philippi ST (2002) há uma diferença na avaliação do consumo alimentar das crianças em diferentes faixas de renda e constatando que o consumo de açúcar é alto em todas elas, porém é maior nas famílias de menor poder aquisitivo.

Segundo a lei no 11.947 de 16 de junho de 2009, e da Resolução FNDE ํㅜㅇ, de 16 de julho de 2009 as escolas públicas passaram a consumir os produtos da agricultura familiar nas refeições oferecidas na escola tais como frutas, hortaliças, bem como alimentos processados/industrializados (queijo, quitandas, leite, conservas e demais produtos panificados) (SCHRÖETTER MR, 2011). Foi Verificado que nas escolas em estudo há o consumo de frutas produzidas pela Agricultura Familiar, sendo que o abacaxi e o mamão apareceram com maior frequência. 
As evidências cientificas mais recentes preconizam o consumo regular de uma quantidade mínima de $400 \mathrm{~g} / \mathrm{dia}$ desses grupos alimentares, para que se tenha uma menor ocorrência para o desenvolvimento de muitas doenças crônicas não-transmissíveis e a manutenção do peso adequado para a idade. Sabe-se que o consumo desse grupo corresponde a três porções diárias (BRASIL, 2014).

Nota-se no estudo em evidência que as verduras ofertadas no cardápio não são adquiridas pela Agricultura Familiar, são, portanto, obtidas por indústrias de alimentos.

O Ministério da Saúde, em suas ações para promoção de uma alimentação saudável, enfatiza a importância da ingestão de verduras, frutas e legumes. Como exemplo podemos citar o Programa Nacional de Agricultura Familiar (Pronaf), do Ministério do Desenvolvimento Agrário, constitui uma oportunidade para aumentar a produção desses alimentos, assegurando a oferta de alimentos frescos e nutritivos em programas institucionais (escolas, creches, asilos, entre outros locais), fazendo a associação entre a produção e o consumo saudável (BRASIL, 2014; BORGES GG, et al., 2018).

Neste estudo encontra-se o grupo de frutas, devendo ser consumido pelo menos (1 porção) e verduras (13 porções). Outro modo bastante comum é o consumo da fruta por meio do suco da polpa, que perde suas propriedades inicial no processo de industrialização (MARTINS CR e FARIAS RM, 2002). A literatura nos traz a relação do consumo das porções da pirâmide alimentar para o escolar com diferentes resultados. $\mathrm{Na}$ pesquisa realizada por Cunha SFC (1998), com pré-escolares, encontrou-se uma ingestão média dos grupos alimentares, por porções/dia: legumes $(1,36)$, frutas $(2,04)$, carnes $(1,79)$, leite $(2,57)$, cereais $(3,16)$ e açúcar $(1,51)$ (ALMEIDA L, et al., 2018). Houve uma inadequação da dieta principalmente no grupo dos legumes, frutas, carnes, leite e cereais. Em pesquisa semelhante realizado por Barbosa RMS et al. (2007), também com pré-escolares, verificou-se inicialmente uma inadequação no consumo dos grupos do leite, legumes, frutas e cereais e após seis meses os resultados demonstraram que os grupos alimentares que não atenderam à recomendação foram os grupos do leite, dos legumes e dos cereais, sendo que o grupo da carne atingiu a porção recomendada em ambos os momentos.

Observou que a média do número de porções consumida ao dia foi de 2,7 para o grupo das frutas e 0,9 para o grupo dos legumes. Crianças latinas consomem em média 2,8 porções de frutas, sucos naturais e vegetais, não atingindo o recomendado pela pirâmide (BARBOSA RMS, et al., 2007). Em relação ao leite e derivados, tem-se a presença de bebida láctea, queijo do tipo muçarela e requeijão cremoso. Analisando o cardápio em relação a ingestão de lácteos, verificou-se que os escolares consomem uma porção de leite/dia. A presença de manteiga de leite é de uma das opções que se apresentam neste consumo diário. Sabe-se que a manteiga é uma das principais fontes de gordura saturada, podendo aumentar o risco de dislipidemias assim como de doenças cardiovasculares. Considerando os pré-escolares ( 1 a 6 anos) e os escolares ( 7 a 14 anos), a quantidade recomendada para atender as necessidades de cálcio requer o consumo de 3 copos (600 ml) e de 2 a 3 copos (400 ml a $600 \mathrm{ml}$ ) de leite no dia (BRASIL, 2014).

Para uma melhor discussão e conclusão acerca deste tema, seriam necessários mais estudos científicos, com intuito de acrescentar mais informações e dados sobre o consumo desses alimentos na merenda escolar. A fim de proporcionar ações de incentivo à produção e ao consumo destes. Ações estas que podem contribuir para a geração de renda dos agricultores e na melhora da qualidade de vida.

\section{CONCLUSÃO}

Considerando a amostra estudada, ressalta-se que o consumo de frutas e verduras é consideravelmente baixo. Provavelmente por questões ligadas a distribuição, o que pode ser adequado com a inclusão em maior frequência de alimentos provenientes da Agricultura Familiar. Ressalta-se a importância de se ter uma fiscalização no sentido de investigar se os alimentos produzidos pela Agricultura Familiar estão sendo distribuídos a todas as escolas, pra atender o que a Política Nacional de Alimentação Escolar preconiza. Neste estudo, observou-se que a alimentação ofertada para crianças das escolas avaliadas não apresenta excesso de alimentos tiocompostos, as cores do cardápio são variadas conforme preconizado, sendo que a oferta de carne gordurosa é baixa. Porém, há uma alta oferta de alimentos que possuem açúcares 
industrializados, sendo pouca a oferta de frutas e verduras, ressaltando-se a necessidade de acompanhamento mais rigoroso na elaboração dos cardápios propostos, para que seja evitado de maneira inerente os possíveis agravos em relação a alimentação inadequada no decorrer de seu desenvolvimento e em sua vida adulta.

\section{REFERÊNCIAS}

1. ALMEIDA L, et al. Consumo de refrigerantes entre adolescentes e o estado nutricional. Revista de Iniciação Científica e Extensão, 2018; 342-346 p.

2. AQUINO RC, PHILIPPI ST. Consumo infantil de alimentos industrializados e renda familiar na cidade de São Paulo. Revista de Saúde Pública, São Paulo, 2002; 655-660 p.

3. BARBOSA RMS, et al. Avaliação da ingestão de nutrientes de crianças de uma creche filantrópica: aplicação do Consumo Dietético de Referência. Revista Brasileira Saúde Materno Infantil, Recife, 2007; 159-166p.

4. BORGES GG, et al. Análise da importância do Pronaf para os agricultores familiares. Revista Expressão Católica, 2018; 20-26 p.

5. CUNHA SFC. Guia Alimentar da pirâmide: experiência em Uberaba, MG. In:Workshop Instituto Danone. Alimentação Equilibrada para a População Brasileira. Florianópolis, 1998.

6. FAO (Food and Agriculture Organization of the United Nations). Alimentar o mundo, cuidar do planeta. Montevideo: FAO, 2014.

7. BRASIL. Guia alimentar para a população brasileira: promovendo a alimentação saudável. Brasília, 2014; 158p.

8. HE FJ, et al. Fruit and vegetable consumption and stroke: meta-analysis of cohort studies. Lancet, London, 2006; 320-326 p.

9. INSTITUTO BRASILEIRO DE GEOGRAFIA E ESTATÍSTICA (IBGE). Pesquisa de Orçamentos Familiares, 20132014. Despesas, Rendimentos e Condições de Vida. Rio de Janeiro: IBGE, 2014.

10. LOCK K, et al. The global burden of disease attributable to low consumption of fruit and vegetables: implications for the global strategy on diet. Bull World Health Organ, Genebra, 2005; 100-108 p.

11. MARTINS CR, FARIAS RM. Produção de alimentos $X$ desperdícios: tipos, causas e como reduzir perdas na produção agrícola - Revisão. Revista da Faculdade de Zootecnia, Veterinária e Agronomia,2002; 83 - 93p.

12. NEUTZLING MB, et al. Fatores associados ao consumo de frutas, legumes e verduras em adultos de uma cidade no Sul do Brasil. Caderno de Saúde Pública, Rio de Janeiro, 2009; 2365-2374 p.

13. OLIVEIRA LG. Avaliação do Programa de Aquisição de Alimentos (PAA) e do Programa Nacional de Alimentação Escolar (PNAE) na agricultura familiar: estudo de caso na microrregião de Ubá-MG. 2015. 168f. Dissertação (Mestrado em Engenharia de Produção), Universidade Federal de São Carlos, São Carlos, 2015.

14. PASSOS ALA. Análise do Cardápio de uma Unidade de Alimentação e Nutrição Institucional em Brasília-DF segundo o método “Avaliação Qualitativa das Preparações do Cardápio”. 2008. 41 f. Monografia (Especialização em Gastronomia e Saúde), Universidade de Brasília, Brasília, 2008.

15. PHILIPPI ST, et al. A. Pirâmide alimentar para crianças de 2 a 3 anos. Revista de Nutrição, Campinas, 2003; 5$19 p$.

16. PROENÇA RPC, et al. Qualidade nutricional e sensorial na produção de refeições. 1. ed. Florianópolis: Editora da UFSC, 2005. 221p.

17. SCHRÖETTER MR. Agricultura familiar e o consumo de alimentos na merenda escolar - o caso da região fronteira noroeste. 2011. 21 f. (Trabalho de Pós-Graduação), Universidade Regional do Noroeste do Estado do Rio Grande do Sul, Santa Rosa, 2011.

18. SILVA EO, et al. Alimentação escolar e constituição de identidades dos escolares: da merenda para pobres ao direito à alimentação. Cadernos Saúde Pública, 2018; 13-25p.

19. SOUZA LCA, et al. Perfil nutricional de pré-escolares do programa mais educação na cidade de Goiânia- GO. Revista de Divulgação Científica Sena Aires, 2019; 36-48p.

20. TEO CRPA, et al. desafios e mecanismos de enfrentamento na relação entre agricultura familiar e alimentação escolar. Revista Grifos, 2017. 34-64p. 